\title{
25 Jahre Akademie für Ethik in der Medizin
}

\author{
Claudia Wiesemann
}

Online publiziert: 5. Juli 2011

(C) Die Autor(en) 2011. Dieser Artikel ist auf Springerlink.com mit Open Access verfügbar.

Mitte der achtziger Jahre des letzten Jahrhunderts: Ich schloss gerade mein Medizinstudium $\mathrm{ab}$ und hätte darüber sehr zufrieden sein müssen. Denn ich hatte ein sehr anspruchsvolles Studium erfolgreich beendet und war dabei, den Beruf mit dem laut Umfragen höchsten Ansehen zu ergreifen. Stattdessen war ich frustriert. Man hatte mich gelehrt, Krankheiten zu identifizieren, aber nicht, mit kranken Menschen umzugehen. Man hatte mir beigebracht, dass Befunde zählen, nicht Wunsch und Wille des Patienten. Wenn in den Vorlesungen und Praktika überhaupt kranke Menschen auftauchten, dann am Rande des Geschehens. Zum ebenbürtigen Gesprächspartner wurden sie allenfalls, wenn es ihnen gelang, sich gemeinsam mit ihren Ärzten als medizinisches Objekt zu betrachten. Freilich gab es auch in dieser Zeit Ärzte, die sich viel Zeit für ihre Patienten nahmen. Wir bewunderten sie. Aber sie taten etwas, wofür es keinen professionellen Grund gab. Es erschien uns wie ein besonders löbliches Hobby.

Wir Studierenden, die wir deprimiert aus einer Patientendemonstration kamen, bei der der Patient weder mit Namen vorgestellt worden war, noch sonst irgendein Wort hatte sagen dürfen, wussten nicht, wie wir unsere Frustration in Worte kleiden sollten. Nicht nur hatte der Patient mit seinem Namen seine Identität, seine Biografie, seine Würde verloren. Es herrschte überhaupt eine existenzielle Sprachlosigkeit für alles, was das Leben als ein Menschliches auszeichnete.

Doch während ich noch darüber grübelte, wie dieser Sprachlosigkeit zu entkommen sei und ob ich je als Ärztin glücklich sein könnte, arbeiteten andere schon an einer systematischen Antwort auf diese Misere der Medizin. Einer so existenziellen Sprachlosigkeit musste man mit einem neuen Vokabular begegnen. Dieses Vokabular entstammte der Ethik. In der Praxis hatte man zwar durchaus versucht, der Unsichtbarkeit des Patienten in der modernen Medizin abzuhelfen. Psychosomatiker stellten die Subjektivität des Leidens in den Vordergrund, Medizinjuristen stärkten die Rechte des Patienten im Medizinbetrieb. Aber indem die moralischen Fragen der Medizin selbst zum Thema gemacht wurden, gelang es am tief-

Prof. Dr. C. Wiesemann ( $₫)$

Abteilung Ethik und Geschichte der Medizin, Universitätsmedizin Göttingen,

Humboldtallee 36, 37073 Göttingen, Deutschland

E-Mail: cwiesem@gwdg.de 
greifendsten, Integrität, Autonomie und Würde des Menschen zum Maßstab ärztlichen Handelns zu erheben. Die moralische Dimension des Problems war nicht mehr nur Anstoß zum Nachdenken, sondern Programm.

Geleitet von dieser Erkenntnis und bestärkt durch Entwicklungen in anderen Ländern, vor allen den USA, traf sich eine Gruppe von Personen, denen die gesellschaftliche und moralische Dimension der Medizin am Herzen lag, und gab dem Kind einen Namen: Ethik in der Medizin. Vorbereitet durch zwei Treffen in der Evangelischen Akademie Loccum fand vor 25 Jahren, am 5./6. Dezember 1986, ein vom Göttinger Medizinhistoriker Prof. Ulrich Tröhler organisiertes, durch das VW-Vorab des Landes Niedersachsen finanziertes „Symposium zur Institutionalisierung von Ethik in der Medizin“"statt. Am 5. Dezember unterschrieb die Mehrheit der Teilnehmer eine Urkunde zur Gründung der Akademie für Ethik in der Medizin.

Es unterzeichneten (in der Reihenfolge, in der sie die Urkunde unterschrieben): der Rechtsmediziner Hans-Bernhard Wuermeling, der Medizinhistoriker Richard Toellner, der Gynäkologe und Herausgeber der Zeitschrift „Arzt und Christ“, Wolfgang Müller-Hartburg, der Medizinhistoriker Ulrich Tröhler, der Internist und Sozialmediziner Helmut Piechowiak, der Internist Hans Jahrmärker, der Medizinhistoriker Eduard Seidler, die Ärztin und Medizinethikerin Gisela Bockenheimer-Lucius, der Psychiater und Sozialmediziner Herbert Viefhues, der Sozialmediziner Hans-Heinrich Raspe, der Internist und Leiter der Gesundheitsabteilung im Ministerium für Umwelt und Gesundheit des Landes Rheinland-Pfalz, Christoph Fuchs, der Chirurg Hans-Konrat Wellmer, die Kinderärztin und Medizinethikerin Bettina Schöne-Seifert, der Arzt und Hochschuldidaktiker Winfried Kahlke, Henning Albrecht vom Stifterverband für die Deutsche Wissenschaft, der Medizinpsychologe Hermann Pohlmeier, der Klinikpfarrer und Studienleiter an der Evangelischen Akademie Loccum, Udo Schlaudraff, sowie der Arzt und Gesundheitssystemforscher Toni Graf-Baumann als Wahlleiter.

Die Dimensionen des damals angestoßenen, ambitionierten Unterfangens werden heute, 25 Jahre später, erkennbar. Medizinethik ist fester Bestandteil der Ausbildung von Ärzten und Pflegenden geworden. Das Fach ist zu einer Spezialdisziplin nicht nur der Medizin, sondern auch - als angewandte Ethik oder Sozialethik - der Philosophie und Theologie geworden. Zahlreiche Menschen engagieren sich unter dem Dach der Akademie für Ethik in der Medizin für einen Dialog zwischen den Disziplinen. Große gesellschaftliche und politische Debatten der Zeit sind von Medizinethikerinnen und Medizinethikern angestoßen worden. Diese Zeitschrift, in der ich zu Ihnen spreche, geht in ihr 24. Erscheinungsjahr und erreicht eine stetig anwachsende Zahl von Leserinnen und Lesern.

Die Medizin selbst hat sich darunter verändert. Ethik ist zu einem selbstverständlichen Thema im klinischen Alltag geworden. Meinen Studierenden muss ich nicht mehr erläutern, warum wir zu ihnen über Patientenrechte sprechen. Das hätte die junge Ärztin von damals nicht zu träumen gewagt!

Open Access Dieser Artikel unterliegt den Bedingungen der Creative Commons Attribution Noncommercial License. Dadurch sind die nichtkommerzielle Nutzung, Verteilung und Reproduktion erlaubt, sofern der/die Originalautor/en und die Quelle angegeben sind. 